\title{
Editorial
}

\section{Embedded-Model-Based Control}

\author{
Sabri Cetinkunt, ${ }^{1}$ Shin-ichi Nakajima, ${ }^{2}$ Brad Nelson, ${ }^{3}$ and Salem Haggag ${ }^{4}$ \\ ${ }^{1}$ Department of Mechanical and Industrial Engineering, University of Illinois at Chicago, Chicago, IL 60607, USA \\ ${ }^{2}$ Department of Mechanical and Control Engineering, Niigata Institute of Technology (NIIT), Niigata 9503315, Japan \\ ${ }^{3}$ Institute of Robotics and Intelligent Systems, ETH Zurich, 8092 Zurich, Switzerland \\ ${ }^{4}$ Faculty of Engineering, Ain Shams University, Cairo 11511, Egypt \\ Correspondence should be addressed to Sabri Cetinkunt; scetin@uic.edu
}

Received 27 June 2013; Accepted 27 June 2013

Copyright (C) 2013 Sabri Cetinkunt et al. This is an open access article distributed under the Creative Commons Attribution License, which permits unrestricted use, distribution, and reproduction in any medium, provided the original work is properly cited.

The industrial practice of mechatronics engineering involves three phases of software development:

Phase 1: control software development and simulation in non-real-time environment;

Phase 2: hardware-in-the-loop (HIL) simulation and testing in real-time environment;

Phase 3: testing and validation on actual machine.

In Phase 1, the control software is developed increasingly by using graphical software tools as opposed to script-based software tools, such as Simulink and Stateflow. Then the developed software is simulated and analyzed on a non-realtime computer environment. The "plant model" which is the computer model of the machine controlled is a non-real-time detailed dynamic model. Simulations and analysis are done in this non-real-time environment.

In Phase 2, the "same control software" is tested on a target embedded control module (ECM). That is, the "same control software" is a C-code which is autogenerated from the graphical diagrams using autocode generation tools. That real-time controller software is run on the target embedded controller module (ECM) hardware which is connected to another computer which simulates the controlled process dynamics in real time. This is called the hardware-in-the-loop (HIL) simulation. This process allows the engineer to test the control software on the actual ECM hardware. The computer which simulates the plant model in real time provides the simulated I/O connections to the ECM. The fundamental challenge in HIL simulations is the to find a balance between the model accuracy (hence, more complex and detailed models) and the need for real-time simulation. As the realtime modeling capabilities are improved, virtual dynamic testing and validation of complete machines using HIL tools will become a reality in engineering design and development processes for embedded control systems. HIL is the testing and validation engineering process between pure software simulation (100\% software) and pure hardware (100\% actual machine) with all its hardware and embedded software, where some of the components are actual hardware and some are simulated in real-time software. The pure softwarebased simulation cannot capture the real-time conditions in sufficient detail to provide the necessary confidence in the overall system functionality and reliability. However, pure hardware testing is quite often too expensive due to the cost of actual hardware, its custom instrumentation for testing purposes, and team of engineers and operators involved in the testing. Furthermore, some tests (especially failure modes) cannot be tested or are very difficult to test (i.e., flight control systems) on the actual hardware.

HIL tools have been developed rapidly in recent years such that some of the hardware components of the control system are included as actual hardware (such as electronic control unit (ECU), engine, transmission, and dynamometer) and some of the components are present in software form running in real time and its results are reflected on the control system by a generic simulator (i.e., dynamometer which represent the load on the powertrain based on the machine dynamics and operating conditions). Early versions of HIL simulations were used to test the static input-output 
behavior of the ECM running the intended real-time control code. Modern HIL simulation and testing are performed for dynamic testing, as well as static testing, where the $\mathrm{I} / \mathrm{O}$ to ECM is driven by dynamic and detailed models of the actual machine.

In Phase 3, the ECM with the control code is tested on the actual prototype machine. First, all of the I/O hardware is verified for proper operation. The sensors and actuators (i.e., solenoid drives and amplifiers) are calibrated. The software logic is tested to make sure all contingencies for fault conditions are taken into account. Then, the control algorithm parameters are tuned to obtain the best possible dynamic performance based on expert operator and end-user comments. The performance and reliability of the machine is tested, compared to benchmark results, and documented in preparation for production release.

In this special edition, we have tried to put together a set of papers to address these essential aspects of the modern mechatronics engineering practice. We hope that it will be a useful collection for our readers.

Sabri Cetinkunt Shin-ichi Nakajima Brad Nelson Salem Haggag 

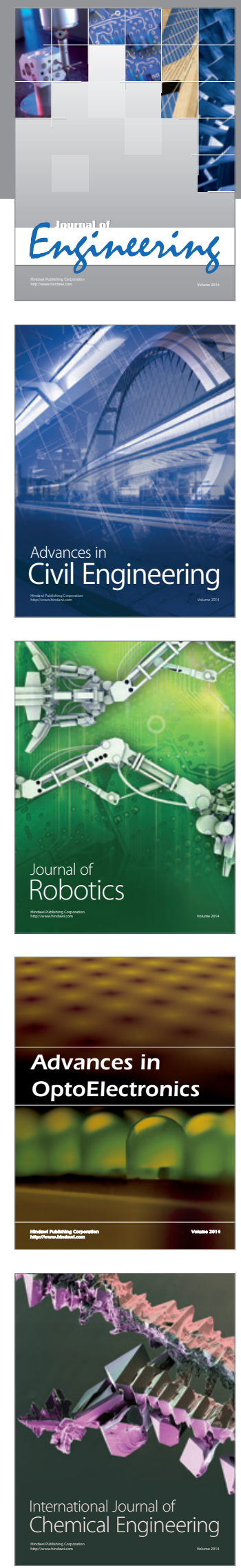

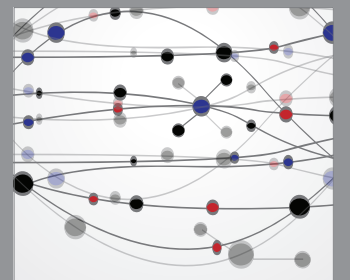

The Scientific World Journal
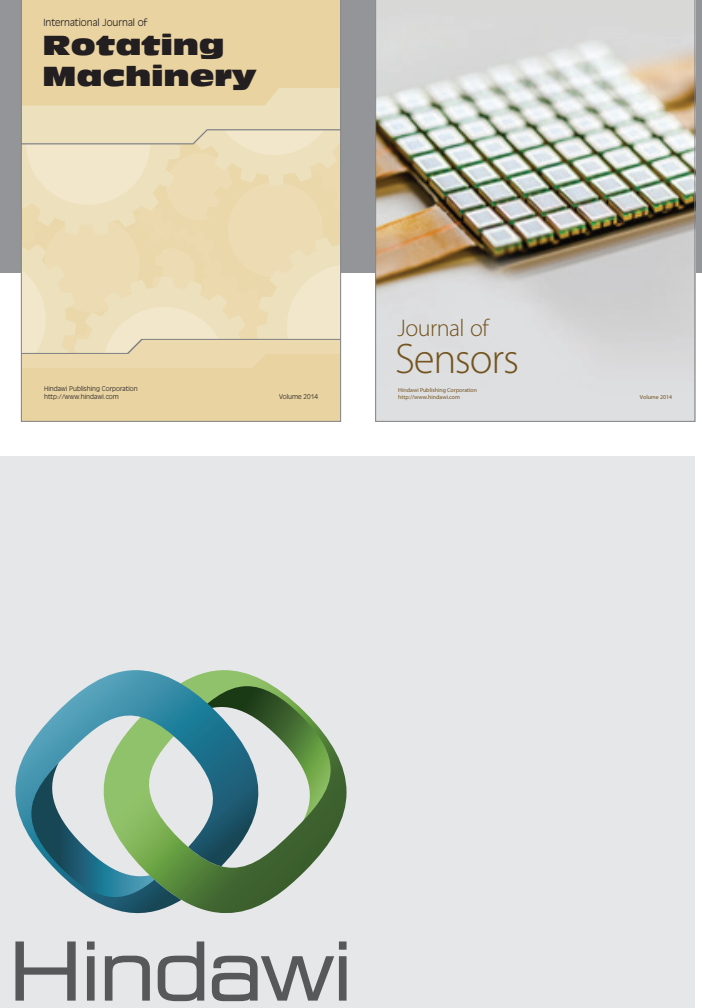

Submit your manuscripts at http://www.hindawi.com
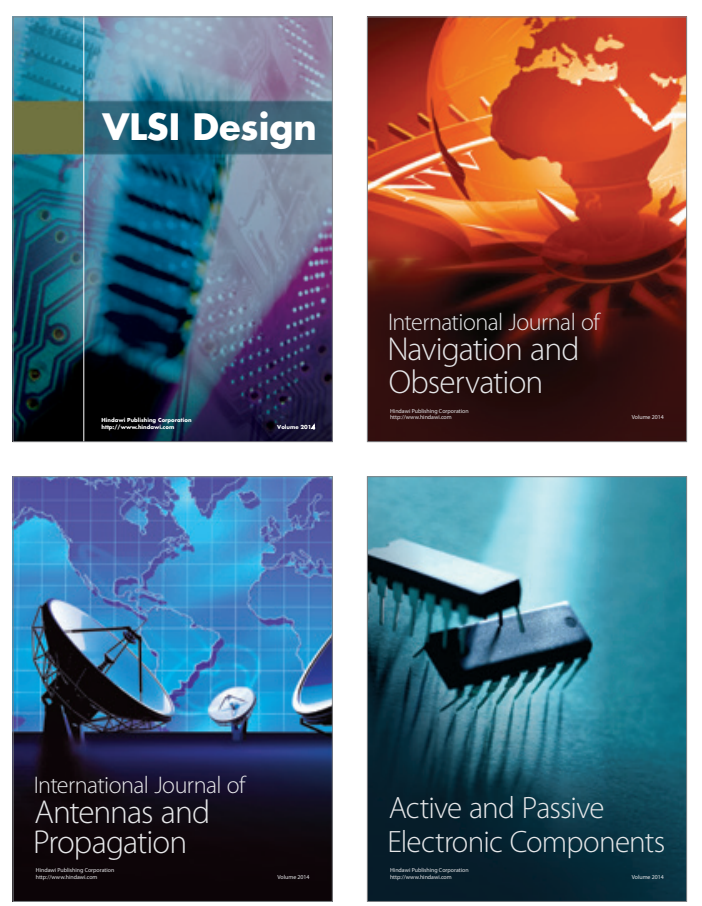
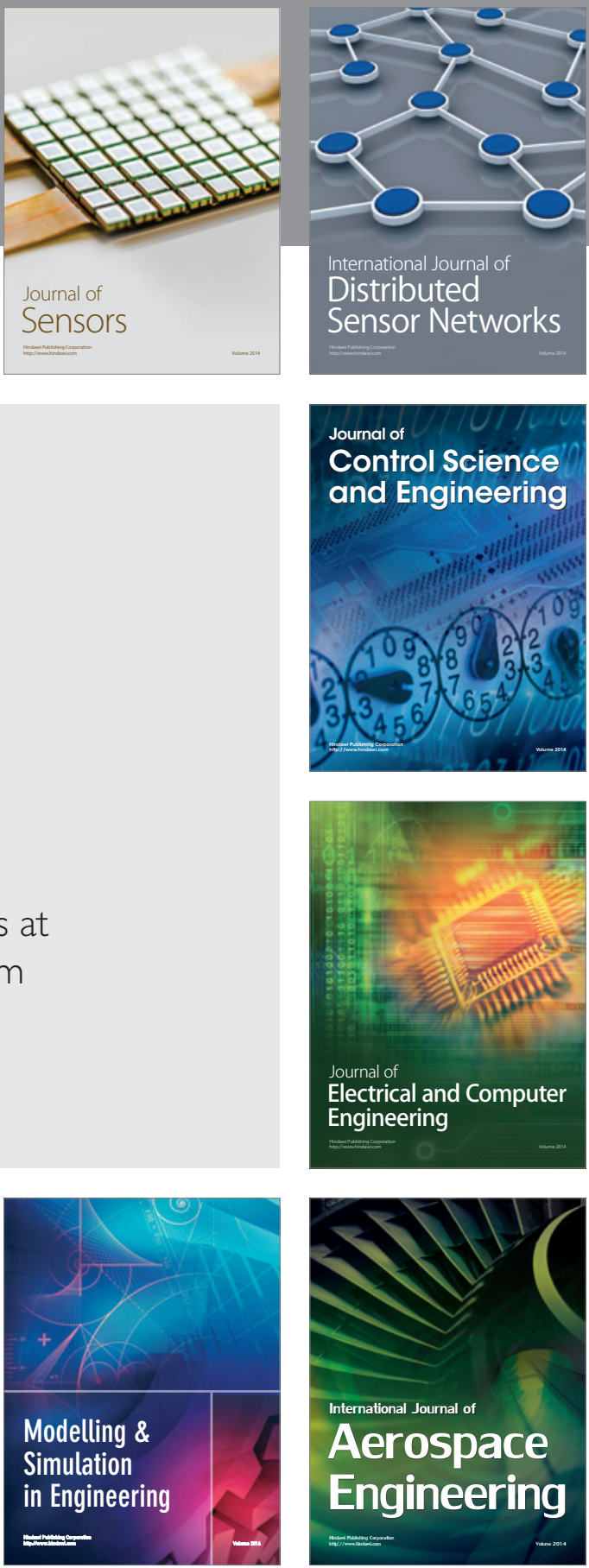

Journal of

Control Science

and Engineering
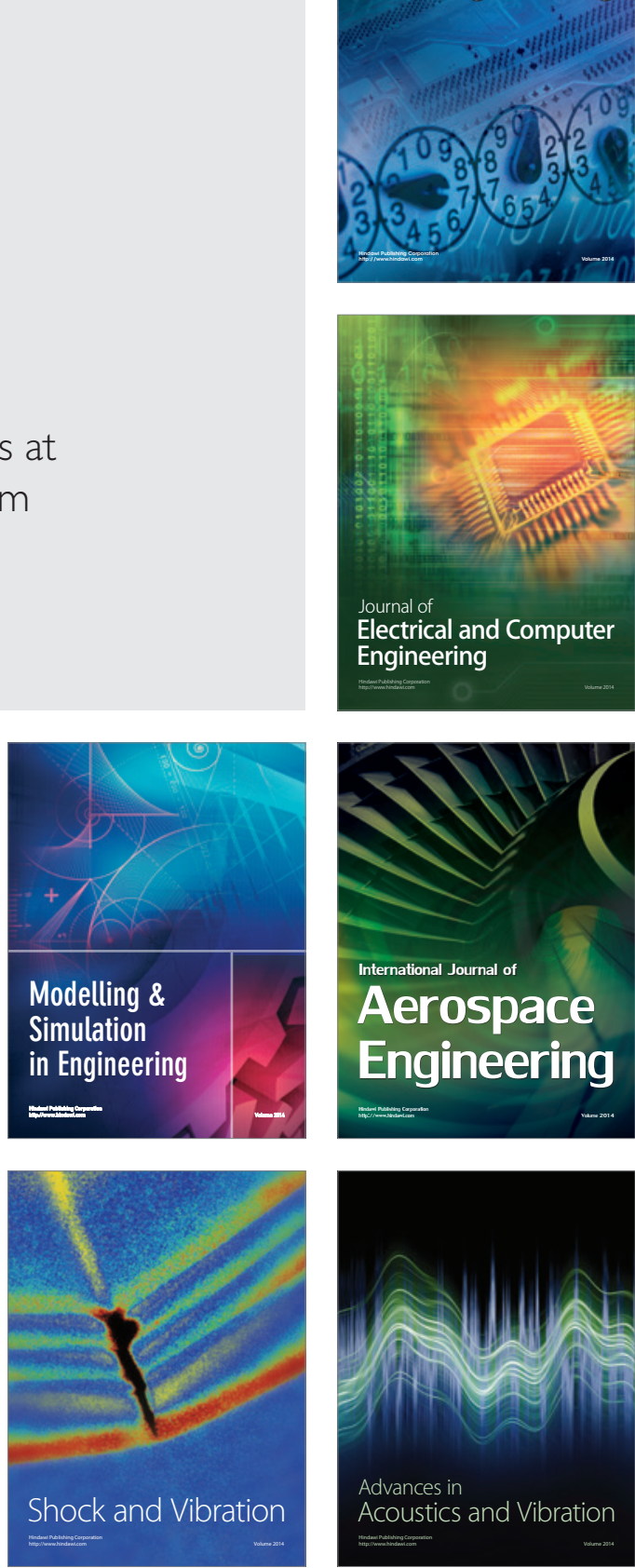